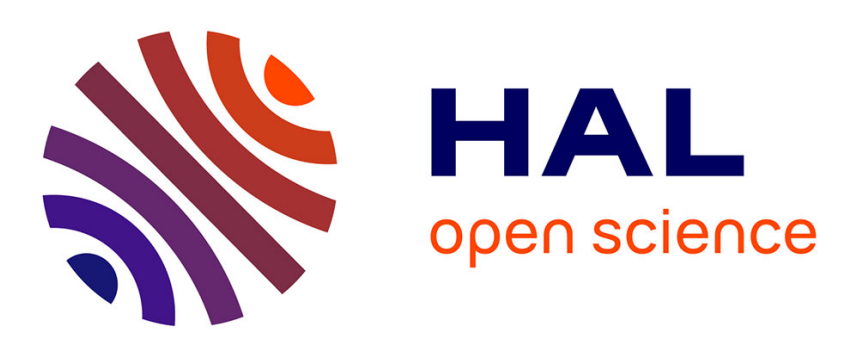

\title{
pycotem: An open source toolbox for online crystal defect characterization from TEM imaging and diffraction
}

Frédéric Mompiou, Rui-xun Xie

\section{- To cite this version:}

Frédéric Mompiou, Rui-xun Xie. pycotem: An open source toolbox for online crystal defect characterization from TEM imaging and diffraction. Journal of Microscopy, 2020, 10.1111/jmi.12982 . hal-03145442

\author{
HAL Id: hal-03145442 \\ https://hal.science/hal-03145442
}

Submitted on 18 Feb 2021

HAL is a multi-disciplinary open access archive for the deposit and dissemination of scientific research documents, whether they are published or not. The documents may come from teaching and research institutions in France or abroad, or from public or private research centers.
L'archive ouverte pluridisciplinaire HAL, est destinée au dépôt et à la diffusion de documents scientifiques de niveau recherche, publiés ou non, émanant des établissements d'enseignement et de recherche français ou étrangers, des laboratoires publics ou privés. 


\title{
pycotem: an open source toolbox for on-line crystal defect characterization from TEM imaging and diffraction
}

\author{
Frédéric Mompiou $^{1 *} \quad$ Rui-Xun Xie ${ }^{2 *}$
}

${ }^{1}$ CEMES-CNRS and Université de Toulouse, Toulouse, France

${ }^{2}$ Key Laboratory of Advanced Materials (MOE), School of Materials Science and Engineering, Tsinghua University, Beijing, People's Republic of China

\section{Correspondence}

CEMES-CNRS, Université de Toulouse, 29 rue J. Marvig, 31055 Toulouse cedex 4,

France

Email: frederic.mompiou@cemes.fr

Funding information
We present a series of tools working together that facilitate the determination of dislocation Burgers vectors and slip planes, interface plane normals, and misorientation between two crystals from a series of TEM micrographs and diffraction patterns. To that purpose, we developed graphical user interface programs that allow crystal orientation determination from spot diffraction patterns taken at various tilt angles or from Kikuchi patterns, crystal representation from stereographic projection plots, and determination of geometrical features from series of conventional images taken at different tilt angles. We present working examples that allow a faster and easier way to analyze data that can especially be retrieved during in-situ straining experiments where dislocations and grain boundaries need to be characterized. More generally, these tools target material scientists interested in daily microstructural characterization in TEM.

\section{KEYWORDS}

crystal orientation, defect analysis, electron diffraction, stereographic projection

Abbreviations: 


\section{INTRODUCTION}

Determining crystal orientation along with the identification of microstructural features (dislocations, interfaces...) is daily work for material scientists using Transmission Electron Microscopy (TEM). This is often performed using conventional microscopy exploiting Bright Field/Dark Field (BF/DF) imaging and Electron Diffraction (ED). Although the procedure to orient a crystal from ED and determine microstructural features from BF/DF is well described in textbooks [1, 2], it is usually a long and technical task. Orientation determination traditionally leans on a two step procedure: i) identify recorded diffraction zone axes from atlases of diffraction patterns or identify the location of a Kikuchi pattern in a map, and ii) determine the crystal directions and planes in the microscope coordinate system. This second task is performed with the help of stereographic projections. Still today, scientists are thus trained to plot them by hand using the Wulff net.

Existing software tools, including packages and standalone programs, mainly simplify these two tasks by proposing the identification of diffraction patterns and by offering a numerical way to plot stereographic projections. We briefly summarize the developed strategies in the following.

ED is a straightforward manner to determine orientation as it collects information on crystal planes and symmetry, either from convergent beam patterns or spot patterns (selected area diffraction or micro/nano-diffraction). When spot patterns are calibrated and taken from zone axes, there are various strategies to automatically index them or help users to do so [3-5]. An alternative way is to compare patterns with calculated ones as proposed in many programs such as JEMS [6], CaRine [7], CrysTBox [5], Landyne [8] or SingleCrystal [9]. This idea has been further exploited to retrieve directly orientation from a spot pattern taken from arbitrary orientations. Various approaches have been proposed in the literature with the culminating objective to perform automated crystal orientation mapping (ACOM) [10-13]. The drawback with ACOM using micro-diffraction is due to the fact that a spot pattern always has 2-fold symmetry that the crystal may not respect, sometimes resulting in crystal orientations with $180^{\circ}$ ambiguity. This effect can be moderated by electron precession [14], but requires additional hardware installed on the microscope.

Using convergent beam ED requires to operate in a TEM mode that does not allow an easy switching with BF/DF imaging for microstructural characterization. Although Kikuchi patterns can be easily obtained in sufficiently thick area, they are usually mixed up with point patterns which prevent an easy automated identification. Recently, Xie et al. [15] have proposed a fast method to orient crystal from Kikuchi lines by manually matching a draggable Kikuchi map in overlay with an experimental pattern. This approach allows to rapidly re-orient a crystal during TEM operation on the condition that the initial orientation has been set up with a zone axis pattern.

Regarding crystal orientation representation, most of the recent software tools, such as KSpace Navigator [16], $\tau$ ompas [15] and ALPHABETA [17], propose stereographic plots to navigate in the reciprocal space by sample tilting. Beside navigation, computer aided stereographic projection plot offers an approach to determine orientation relationships [18] or to predict crystallographic features related to phase transformation, such as habit plane or dislocation content [19].

Defect analysis, such as dislocations or interface/grain boundaries, is usually guided by stereographic projections and will force the user to tilt the specimen to different diffraction conditions. For example, the Burgers vector of a dislocation is often identified by the $\boldsymbol{g} \cdot \boldsymbol{b}$ invisibility criterion, which needs multiple diffraction conditions. This can even be performed during in-situ experiments [20], as long as preplanned with stereographic projections. Analyzing interface planes or dislocation slip planes also requires a lot of sample tilting. Though algorithms were developed [21-23], not many software tools were reported on this purpose. $\tau$ ompas [15] offers a user-friendly tool to identify the plane from its projections, but a priori guess of plane indices is still needed.

Although a lot of software is available, few of them were reported to offer the ability to determine orientation 
from raw data and analyze defects from the combined information of imaging and diffraction. This often requires switching between different tools, exporting/importing data and normalizing data convention, which are tedious and may become pitfalls.

Here we present an open toolkit, pycotem, that aims to an efficient workflow for crystal orientation analysis and the determination of defects such as dislocations and interface planes. Six different independent tools, with userfriendly graphical interfaces, are provided to orient a crystal with ED (diffraction and kikuchi), determine plane or interface normals or directions from a series of BF/DF images (interface), perform crystallographic analysis of one or two crystals on a stereographic projection (steroproj and misorientation), and finally view the atomic structure on a given projection plane (crystal). steroproj is the core tool to display and interpret crystal orientation and features determined from the other components. The tool diffraction proposes a new general algorithm to determine and refine the crystal orientation from a series of spot patterns recorded at arbitrary conditions. We recently proposed a general method to determine interface normal from a set of BF/DF images with a good accuracy [23]. This method, previously in command line form, is implemented in interface to perform graphical determination of lines or planes. The present paper will not cover all the features proposed by pycotem, but readers can refer to the complete documentation [24]. It is thus organized as follows: we first describe the framework and the method used to determine crystal orientation from spot patterns and interface planes/directions from images. Then, we illustrate this approach with working examples.

\section{2 | METHODS}

\subsection{Crystal orientation definition}

The crystal orientation can be specified by a rotation, or a combination of rotations, which relates the crystal lattice coordinate system to the fixed coordinate system of the microscope, hereafter noted by the subscripts $c$ and $M$, respectively. There are several ways to define the orientation. The classical one refers to the definition of Bunge [25] using a sequence of three rotation of angles $\left(\varphi_{1}, \phi, \varphi_{2}\right)$, the so-called Euler angles. If we define the Euler angles from extrinsic rotations, i.e. rotations that operate in the fixed reference frame $(x, y, z)_{M}$, this corresponds to: a rotation along $z$ of an angle $\varphi_{2}$, a rotation along $\boldsymbol{x}$ of $\phi$, and a rotation along $\boldsymbol{z}$ of $\varphi_{1}$, i.e in terms of rotation matrix:

$$
\boldsymbol{R}=\boldsymbol{R}_{\mathrm{Z}}\left(\varphi_{1}\right) \boldsymbol{R}_{\mathrm{x}}(\phi) \boldsymbol{R}_{\mathrm{Z}}\left(\varphi_{2}\right)
$$

where $\boldsymbol{R}_{\mathrm{Z}}(\theta)$ and $\boldsymbol{R}_{\mathrm{x}}(\theta)$ are the rotation matrices along $\boldsymbol{x}$ and $\boldsymbol{z}$ axis of an angle $\theta$. Their expression is recalled in the Appendix. Inversely, knowing the rotation matrix, the Euler angles can be determined with elements in the matrix:

$$
\varphi_{1}=\arctan \left(\boldsymbol{R}_{13} / \boldsymbol{R}_{23}\right), \phi=\arccos \left(\boldsymbol{R}_{33}\right), \varphi_{2}=\arctan \left(\boldsymbol{R}_{31} / \boldsymbol{R}_{32}\right)
$$

The orientation is thus defined by:

$$
[x, y, z]_{M}^{\top}=\boldsymbol{R}_{\varphi_{1}, \phi, \varphi_{2}}[x, y, z]_{\mathrm{C}}^{\top}
$$

with the ${ }^{\top}$ sign being for transpose, indicating column vectors. For a given crystal, the direction $[u, v, w]$ or plane $(h, k, l)$ are related to $[x, y, z]$ by: 


$$
[x, y, z]_{\mathrm{C}}^{\top}=D[u, v, w]_{\mathrm{C}}^{\top}=\left(D^{-1}\right)^{\top}(h, k, l)_{\mathrm{C}}^{\top}
$$

where:

$$
\boldsymbol{D}=\left(\begin{array}{ccc}
a & b \cos \gamma & c \cos \beta \\
0 & b \sin \gamma & c(\cos \alpha-\cos \beta \cos \gamma) / \sin \gamma \\
0 & 0 & V /(a b \sin \gamma)
\end{array}\right)
$$

and $a, b, c, \alpha, \beta, \gamma$ the crystal parameters, and $V$ the crystal volume.

So that the orientation is determined by:

$$
[x, y, z]_{\mathrm{M}}^{\top}=\boldsymbol{R}_{\varphi_{1}, \phi, \varphi_{2}} D[u, v, w]_{\mathrm{C}}^{\top}=\boldsymbol{R}_{\varphi_{1}, \phi, \varphi_{2}}\left(D^{-1}\right)^{\top}(h, k, l)_{\mathrm{C}}^{\top}
$$

When determining $\boldsymbol{R}_{\varphi_{1}, \phi, \varphi_{2}}$ with ED, the orientation is determined from $\mathrm{N}$ inputs by:

$$
\boldsymbol{R}_{\varphi_{1}, \phi, \varphi_{2}}\left(\boldsymbol{D}^{-1}\right)^{\top}\left(\begin{array}{ccc}
h_{1} & \ldots & h_{N} \\
k_{1} & \ldots & k_{N} \\
I_{1} & \ldots & I_{N}
\end{array}\right)_{C}=\left(\begin{array}{ccc}
x_{1} & \ldots & x_{N} \\
y_{1} & \ldots & y_{N} \\
z_{1} & \ldots & z_{N}
\end{array}\right)_{M}
$$

In Eq.3, $\left[x_{i}, y_{i}, z_{i}\right]_{M}^{\top}$ needs to be measured from patterns of ED, and their corresponding $\left(h_{i}, k_{i}, l_{i}\right)_{\mathrm{C}}^{\mathrm{T}}$ needs to be assigned. There are two sources of $\left[x_{i}, y_{i}, z_{i}\right]_{M}^{\top}$ : a single Kikuchi pattern, or a series of spot patterns recorded at different tilting positions. In the following we will present these two approaches to get $\left[x_{i}, y_{i}, z_{i}\right]_{\mathrm{M}}^{\top}$, then index them with $\left(h_{i}, k_{i}, l_{i}\right)_{\mathrm{C}}^{\mathrm{T}}$, and finally describe the method to retrieve $\boldsymbol{R}_{\varphi_{1}, \phi, \varphi_{2}}$ when Eq.3 is over-determined.

\subsection{Determining the diffraction vectors in microscope coordinates}

\subsection{1 | Coordinate system of the projection screen}

To reach a desired diffraction condition, the specimen must be tilted in TEM. Usual specimen holders allow tilting along a primary axis, called $\alpha$-tilt, fixed in the microscope coordinate system and a second tilt, called $\beta$-tilt (double tilt holder), or $\theta$-tilt (tilt-rotation holder), that moves in the microscope coordinate system. In pycotem, $\alpha$-tilt is oriented along $\boldsymbol{y}, \beta$-tilt along $\boldsymbol{x}$ and $\theta$ tilt along $\boldsymbol{z}$ (fig. 1a). Because of magnetic rotation in the electron lenses, the recorded diffraction pattern on the viewing screen or camera is often rotated. The coordinate system in the projected screen noted with the subscript $\mathrm{p}$ is then rotated with respect to the microscope coordinate system by an angle $\xi$ along the $z$ direction (fig. 1b-c).

Giving the tilt angles $\alpha, \beta^{1}$, and the angle $\xi$, the transformation from projection screen coordinates to microscope

\footnotetext{
${ }^{1}$ for the sake of simplicity the equations in the following are expressed for a couple $(\alpha, \beta)$ but also apply for a tilt sequence $(\alpha, \theta)$
} 
coordinates is:

$$
[x, y, z]_{\mathrm{M}}^{\top}=\boldsymbol{R}_{\mathrm{x}}(-\beta) \boldsymbol{R}_{\mathrm{y}}(-\alpha) \boldsymbol{R}_{\mathrm{z}}(\xi)[x, y, z]_{\mathrm{P}}^{\top}
$$

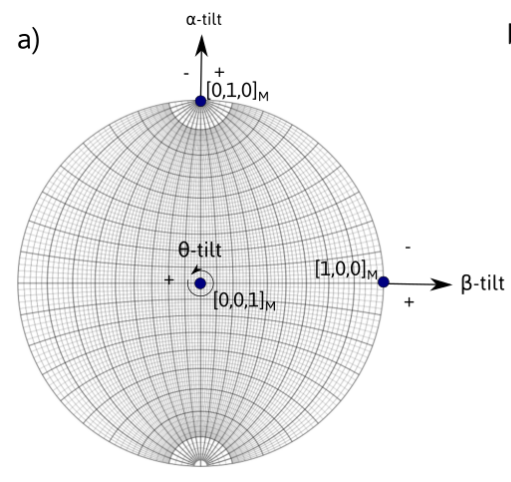

b)

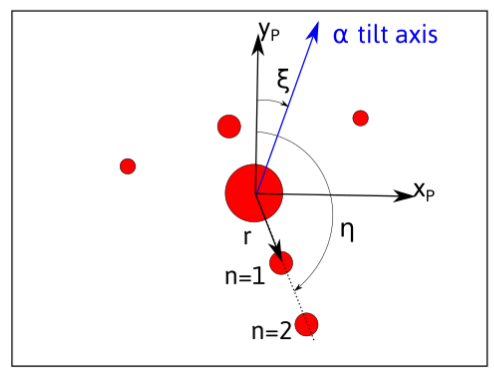

c)

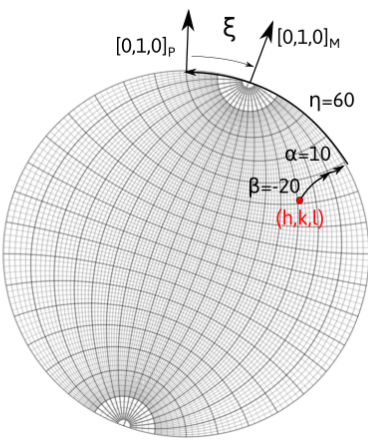

FIGURE 1 a) Fixed coordinate system of the microscope displayed on a Wulff net. The sample can be rotated along the primary $\alpha$-tilt axis and additionally along the $\beta$ or $\theta$ tilt. b) Sketch of a diffraction pattern showing a systematic diffraction row, distant of $r$ from the transmitted beam and at an angle $\eta$ with the $y_{\mathrm{P}}$ axis in the projected screen coordinate system. $n$ denotes the diffraction order. In this coordinate system, the $\alpha$ tilt axis makes an angle $\xi$ with $y_{\mathrm{P}}=[0,1,0]_{\mathrm{P}}$. c) Representation on the Wulff net of a tilt sequence $(\alpha, \beta)$ needed to bring the $(h, k, l)$ reflection at the inclination $\eta$ in the projected screen coordinate system.

\subsubsection{Diffraction vectors from a series of spot patterns}

In a spot pattern, the diffraction vector is defined by the vector from the transmission spot to the diffraction spot. Since the deviation between three-beam conditions $(0,-\boldsymbol{g},+\boldsymbol{g})$ and two-beam conditions $(0,+\boldsymbol{g})$ is usually within a few tenths of a degree, e.g. $0.4^{\circ}$ for (200) of iron austenite at $200 \mathrm{kV}$, which is much smaller than the error introduced by the inaccuracy of the sample holder (see below), we assume in the following that the g-vectors are always on the projection screen ${ }^{2}$. Once the inclination angle $\eta$ of a diffraction vector is retrieved (fig. $1 \mathrm{~b}$ ), its coordinates in projection screen can be expressed as:

$$
[x, y, z]_{\mathrm{P}}^{\top}=\boldsymbol{R}_{\mathrm{Z}}(-\eta)[0,1,0]_{\mathrm{P}}^{\top}
$$

With Eq.4 and Eq.5, the direction of one diffraction vector can be placed on the stereographic projection. This however is not sufficient to derive the crystal orientation as it requires the knowledge of an extra rotation along the vector $[x, y, z]_{\mathrm{M}}$. Thus, once another reflection is determined at the same or a different couple $(\alpha, \beta)$, the orientation can be determined. Working graphically, the orientation in pycotem can be plotted on the stereographic projection by fixing one reflection and rotating around it until the other reflection matches.

\footnotetext{
${ }^{2}$ This is exact if the diffraction pattern is taken in the middle of the extinction contour, i.e. for a deviation from Bragg angle $\mathrm{s}=-\lambda / 2\|\boldsymbol{g}\|^{2}$ (three beam condition). If $s$ is measured, from the position of the Kikuchi bands, the exact coordinates in the plane can be corrected for a given two beam condition by modifying equation 5 by $[x, y, z]_{\mathrm{P}}^{\top}=\boldsymbol{R}_{\mathrm{Z}}(-\eta) \boldsymbol{R}_{\mathrm{X}}(\epsilon)[0,1,0]_{\mathrm{P}}^{\top}$ with $\epsilon=\mathrm{s} /\|\boldsymbol{g}\|+\theta_{B}$. In pycotem, we simply propose an option to correct from a two-beam to a three-beam conditions i.e. taking $\epsilon=\theta_{B}$.
} 
This approach can be easily performed for diffraction patterns recorded during a single tilt experiment, but become difficult in a general case where tilt axes are different. Due to experimental errors, it is also impossible for the operator to align more than 2 reflections altogether with a good accuracy. In part 2.4, we propose a method that avoids any graphical approach or any manual adjustment.

\subsection{3 | Diffraction vectors from a Kikuchi pattern}

A Kikuchi pattern recorded at a given tilting condition present a series of bands that can be defined using 3 points. Two points along one line (excess or defected) define the azimuth angle $\eta$ with respect to the $y_{p}$ axis in the observation plane. A third point taken on the second line defines the projected band width $d_{\mathrm{p}}$ in the observation plane, which is needed to find the center position of the band. A fourth point defines the pattern center $O$. All these points define the band normal projected vector $\boldsymbol{v}_{\mathrm{p}}$, as shown in fig. 2 .

The band normal vectors in the coordinate system $[x, y, z]_{\mathrm{p}}$ are inclined of an angle $\phi$ with respect to the projection plane. They can be derived from the relation:

$$
[x, y, z]_{\mathrm{P}}=\boldsymbol{v}_{\mathrm{p}}+\left[0,0,\left\|\boldsymbol{v}_{\mathrm{p}}\right\|^{2} / L\right]
$$

where $L=\|O P\|$ is the camera length.

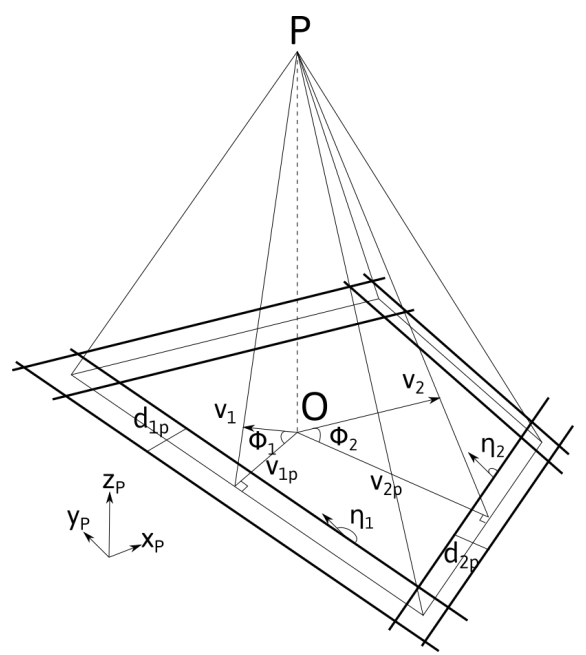

FIGURE 2 Description of a Kikuchi pattern in the $[x, y, z]_{\mathrm{P}}$ coordinate system.

\section{3 | Indexing diffraction vectors with crystal coordinates}

Two strategies can be exploited to determine the correct $(h, k, l)$ indices of diffraction vectors. The first one is to use $\mathrm{d}$-spacing to identify the family, $\{h, k, I\}$, of g-vectors. This can be done by computer on spot patterns recorded with strong reflections. Thus, the observed g-vectors can be restricted to a small set of diffraction vectors $\left(h_{i}, k_{i}, l_{i}\right)$ within some uncertainty range. While the indices of the first one can be chosen arbitrarily, the following ones need to be 
chosen within a set that respect the measured angles $\theta_{i j}=\angle\left(\left[x_{i}, y_{i}, z_{i}\right]_{M},\left[x_{j}, y_{j}, z_{j}\right]_{M}\right)$. The difference between the measured angles and the theoretical ones $\theta_{i j}^{\prime}=\angle\left(\left(h_{i}, k_{i}, l_{i}\right),\left(h_{j}, k_{j}, l_{j}\right)\right)$ can be displayed as an help to discriminate the correct set of indices.

When the g-vectors are ambiguous to discriminate from the sole measure of their d-spacing, which is often the case when working with Kikuchi bands of high indices, the entire sets of g-vectors are those for which all the differences $\left|\theta_{i j}-\theta_{i j}^{\prime}\right|$ are below a tolerance angle. This can be achieved by inspecting a lookup table of angles $\theta_{i j}^{\prime}$. With standard recent PC, this can be achieved within few seconds when considering up to 6 reflections with indices up to the fifth order.

\section{4 | Orientation determination}

In Eq.3, $\boldsymbol{R}_{\varphi_{1}, \phi, \varphi_{2}}$ is a rotation matrix that needs to be determined. When the number of inputs $\mathrm{N}$ is equal to or more than 2, Eq.3 will become a determined or overdetermined equation like:

$$
\boldsymbol{R}_{\varphi_{1}, \phi, \varphi_{2}}\left(D^{-1}\right)^{\top} G_{\mathrm{C}}=G_{\mathrm{M}}
$$

Finding the closest orientation matrix $R_{\varphi_{1}, \phi, \varphi_{2}}$ from Eq.7 can be done with two equivalent approaches proposed by Mackenzie [26] and Horn [27]. Here we follow Mackenzie's least-squares method, which consists in maximizing the sum of cosine of angular deviation defined as:

$$
S=\sum_{r}^{m} \cos \theta_{r}=\sum_{r}^{m}\left(x_{r}, y_{r}, z_{r}\right)_{\mathrm{M}} \boldsymbol{R}\left(\boldsymbol{D}^{-1}\right)^{\top}\left(h_{r}, k_{r}, I_{r}\right)_{\mathrm{C}}^{\top}
$$

Then singular value decomposition is applied to find the $\boldsymbol{R}$ that maximizes $S$ :

$$
\begin{array}{r}
\boldsymbol{U} \wedge \boldsymbol{V}^{\top}=\operatorname{svd}\left(\left(D^{-1}\right)^{T} \boldsymbol{G}_{\mathrm{C}} \boldsymbol{G}_{\mathrm{M}}^{\top}\right) \\
\boldsymbol{R}_{\varphi_{1}, \phi, \varphi_{2}}=\boldsymbol{V} \boldsymbol{U}^{\top}
\end{array}
$$

To test the robustness of the results from experimental errors, the following statistical approach was carried out. From a given orientation matrix $\boldsymbol{R}$, a set of $\mathrm{N}$ g-vectors $(h, k, l)$, ranging from 2 to 7 , were taken. In the microscope coordinate system, vectors corresponding to these g-vectors are computed, added with some error, and used to compute back the orientation matrix $\boldsymbol{R}_{d}$. In the first case simulating spot patterns, the $(\alpha, \beta)$ position of patterns and the inclination angle of spots have random errors ranging between 1 and 3 degrees. This range is expected to account for deviation from the Bragg conditions (usually few tenths of a degree), inclination $\eta$ measurement error and goniometer backlash and inaccuracy. They lead to the colored curves in fig. 3a. In the second case simulating Kikuchi patterns, a random error on the azimuth angle $\eta$ of the line, ranging between 1 and 3 degrees, was introduced corresponding to the 3 colored curved in figure $3 \mathrm{~b}$. A random error ranging between 2.5 and $10 \%$ on the measurement on the distance between the pattern center and the middle of the Kikuchi band, was also introduced and lead to the error bars in fig. $3 \mathrm{~b}$.

The angular deviation between $\boldsymbol{R}$ and $\boldsymbol{R}_{d}$ is given by: 


$$
\theta_{d}=\arccos \left(\frac{\operatorname{tr}\left(\boldsymbol{R} \boldsymbol{R}_{d}^{\top}\right)-1}{2}\right)
$$

The calculations were ran 10000 times which allow to compute a statistical significant average angular deviation. Figure 3 shows the mean angular deviation $\left\langle\theta_{d}\right\rangle$ with respect to the number of diffraction vectors $N$ for different errors.

In both cases, the average error between the expected orientation and the measured one decreases with the number of inputs. Hence in typical tilt series experiments where more than 4 diffraction vectors are obtained, the error is of the order of $1^{\circ}$. For Kikuchi patterns, the error can be reduced down to few tenth of degrees. The main error is due to imprecision in the position of the lines in the observation plane more than the inclination of the diffraction vectors out of the plane. It should be noted that a systematic error can also be introduced if the camera length is not exactly calibrated. This can be however refined by iteratively changing the camera length until a minimum angular deviation is found (see the workflow example in sec. 3).
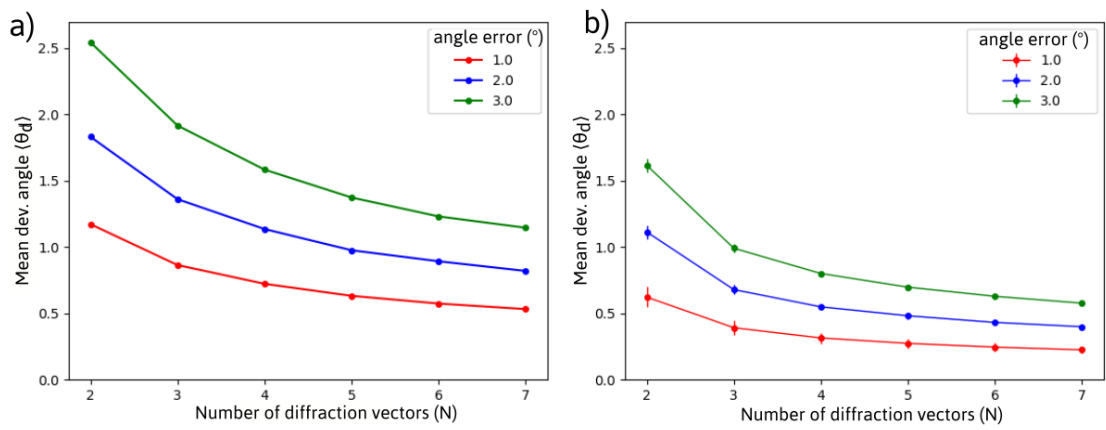

FIGURE 3 Mean deviation angle computed from a random erroneous data sets as a function of number of diffraction vectors for a series of diffraction patterns in a) and a Kikuchi pattern in b).

\subsection{Plane determination from a set of images}

A similar approach can be performed to determine direction or plane normal from a set of images. It is described in more details in [23].

For a given orientation determined by the beam direction $b_{e}$ in the microscope coordinate system, the interface plane $\boldsymbol{n}$ is fully described by the direction of the projected traces $\boldsymbol{t}_{p}$ that correspond to the intersection between the foil surfaces $s$ and the plane. In the projection screen coordinates, the direction of the trace can be characterized by the azimuth angle $\eta$ with the $y_{\mathrm{P}}$ direction. Note that because of the electron optics, the projected plane frame can be rotated with respect to the microscope coordinate system along $z_{\mathrm{M}}=z_{\mathrm{P}}$ of an angle $\delta$ (which can be different from the angle $\xi$ in diffraction mode).

If the two surfaces are parallel, the two projected traces are also parallel (fig. 4). Thus, the width $w$ between the two traces can be also measured. These data constitute the set $\left(b_{\mathrm{e}}, \eta, w\right)$. If the surfaces are not parallel, the method can also be applied owing tracking a specific known point at the interface [23].

It can be shown that the apparent width $w$ is given by: 


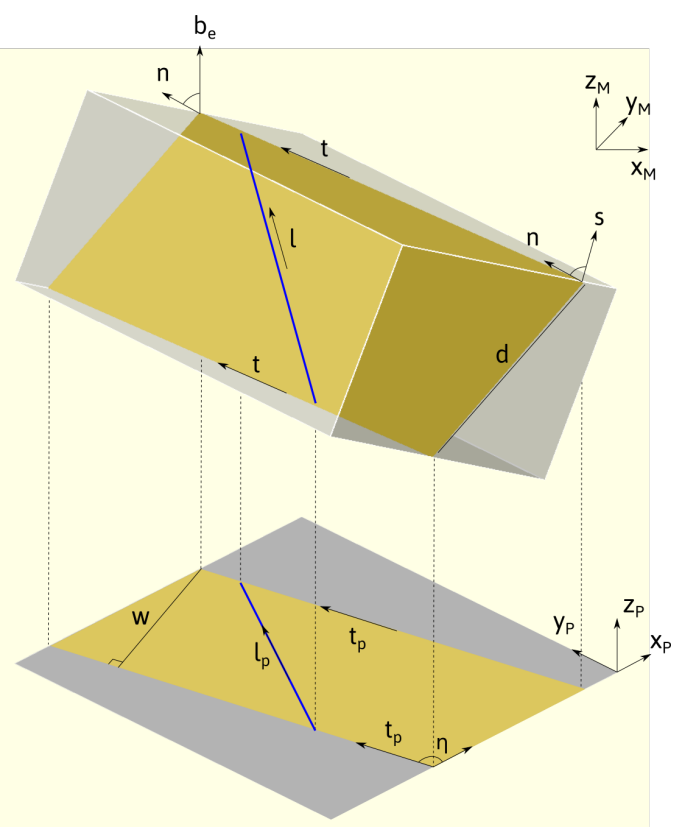

FIGURE 4 Sketch showing the geometry of planar and linear feature in a foil and projected in the viewing screen. The knowledge of the beam direction $b_{\mathrm{e}}$, projected line $\boldsymbol{l}_{\mathrm{p}}$ or trace $\boldsymbol{t}_{\mathrm{p}}$ directions and projected plane width $w$ or line length $\left\|\boldsymbol{l}_{\mathrm{p}}\right\|$, at different tilts allow the determination of plane normal $\boldsymbol{n}$ or line direction $\boldsymbol{l}$.

$$
w=\frac{\left|d \boldsymbol{n} \cdot \boldsymbol{b}_{\mathrm{e}}\right|}{\sqrt{1-\left(\boldsymbol{b}_{\mathrm{e}} \cdot \boldsymbol{t}\right)^{2}}}
$$

where $d$ is the plane width.

The unit normal vector $\boldsymbol{n}$ can be estimated following the two steps procedure. First the trace direction $\boldsymbol{t}$ is solved from a set of $N$ imaging conditions:

$$
\left[b_{\mathrm{e} 1} \times t_{\mathrm{p} 1}, \ldots, b_{\mathrm{e} m} \times t_{\mathrm{p} m}\right]^{\top} \cdot t=0
$$

then the interface normal is solved:

$$
\left[\boldsymbol{b}_{\mathrm{e} 1}^{\top}, \ldots, \boldsymbol{b}_{\mathrm{e} N}^{\top}, t^{\top}\right]^{\top}(d \boldsymbol{n})=\left[\operatorname{sgn}(1) w_{1} \sqrt{1-\left(\boldsymbol{b}_{\mathrm{e} 1} \cdot \boldsymbol{t}\right)^{2}}, \ldots, \operatorname{sgn}(N) w_{N} \sqrt{1-\left(\boldsymbol{b}_{\mathrm{e} N} \cdot \boldsymbol{t}\right)^{2}}, 0\right]^{\top}
$$

the $\operatorname{sgn}(i)$ here denotes the sign of dot product $\boldsymbol{b}_{\mathrm{e} i} \cdot \boldsymbol{n}$. As the projected width $w_{i}$ is always a positive value, but $\boldsymbol{b}_{\mathrm{e} i} \cdot \boldsymbol{n}$ may be positive or not, the sgn $(i)$ is undetermined. The equation above is solved by considering all possible combinations of $\operatorname{sgn}(i)$. The most consistent solution corresponds to the combination leading to the smallest residual error. 
The inputs data used can be derived from the position of the beam direction and apparent trace direction in the microscope coordinate system owing the tilt angles:

$$
\begin{array}{r}
\boldsymbol{b}_{\mathrm{e} i}=\boldsymbol{R}_{\mathrm{x}}\left(-\beta_{i}\right) \boldsymbol{R}_{\mathrm{y}}\left(-\alpha_{i}\right)[0,0,1]_{\mathrm{P}}^{\mathrm{T}} \\
\boldsymbol{t}_{\mathrm{p} i}=\boldsymbol{R}_{\mathrm{x}}\left(-\beta_{i}\right) \boldsymbol{R}_{\mathrm{y}}\left(-\alpha_{i}\right) \boldsymbol{R}_{\mathrm{z}}(\delta) \boldsymbol{R}_{\mathrm{Z}}(\eta)[0,1,0]_{\mathrm{P}}^{\top}
\end{array}
$$

The same but simpler approach can be used to determine a linear feature $\boldsymbol{l}$. Using the data sets $\left(\boldsymbol{b}_{\mathrm{e} i}, \boldsymbol{l}_{\mathrm{p} i}, l_{i}\right)$, where $l_{i}$ is the projected line length, $l$ can be deduced by solving :

$$
\left[\boldsymbol{b}_{\mathrm{e} i} \times \boldsymbol{l}_{\mathrm{p} i}, \boldsymbol{l}_{\mathrm{p} i}\right]^{\top} d \boldsymbol{l}=\left[0, \operatorname{sgn}(i) l_{i}\right]^{\top}
$$

A similar statistical treatment as performed above, shows also that the average deviation angle between an erroneous set of data and a chosen plane normal is of the order of few degrees and decreases with increasing the number of data sets, with typical values of 1 degree for 6 datasets [23].

Without knowing the true normal or direction, the accuracy of a given set of data can be estimated using the bootstrap method [28]. Starting from the set of data $\left(\boldsymbol{b}_{\mathrm{e} i}, \eta_{i}\right)$ or $\left(\boldsymbol{b}_{\mathrm{e} i}, w_{i}\right)$, to estimate the trace direction and normal, respectively, the data sets were resampled and estimated traces and normals were determined using the equations above. The $92 \%$ confidence interval was calculated from the 95 th percentile of deviation of the trace and normal. This indicates that the normal has a large chance to locate in a cone centered around $\boldsymbol{n}$ with a deviation of $\Delta \theta=\angle\left(\boldsymbol{n}, \boldsymbol{n}_{0.95}^{\prime}\right)$.

\section{3 | PYCOTEM WORK-FLOW EXAMPLES}

We propose here to describe the workflow used in pycotem in order to determine orientations and analyze defects. The code, written in python (version 3), is available under the GNU GPLv3 license at github and on the python package repository [29]. Binary executable files for Windows operating systems can also be downloaded.

\section{1 | Orientation of a crystal}

\subsection{1 | from spot patterns}

The tool diffraction allows to determine the orientation from a set of diffraction patterns with the method described above.

It relies on a procedure consisting first in recording diffraction patterns, preferably two-beam ones, at arbitrary tilt conditions. This can be done by varying the tilt angles in image mode and by recording selected area or micro/nanodiffraction patterns when the bending contours crosses the area of interest. As at least two patterns are needed, this procedure can be performed quite fast without looking specifically at zone axes patterns.

Once the patterns are recorded, the tool diffraction can be used. As the first step, the crystal lattice needs to be set (fig. 5a). Then, one can load a diffraction pattern and set its calibration. The $d$-spacing and the inclination angle $\eta$ of diffraction vectors can be retrieved by clicking the transmission spot and diffraction spots successively (fig. 5a). When using a computer aided determination, a list of g-vectors within a tolerance range are proposed in the result panel (fig. 5b). In order to help the user to choose the proper indices, these g-vectors are presented with kinematic 


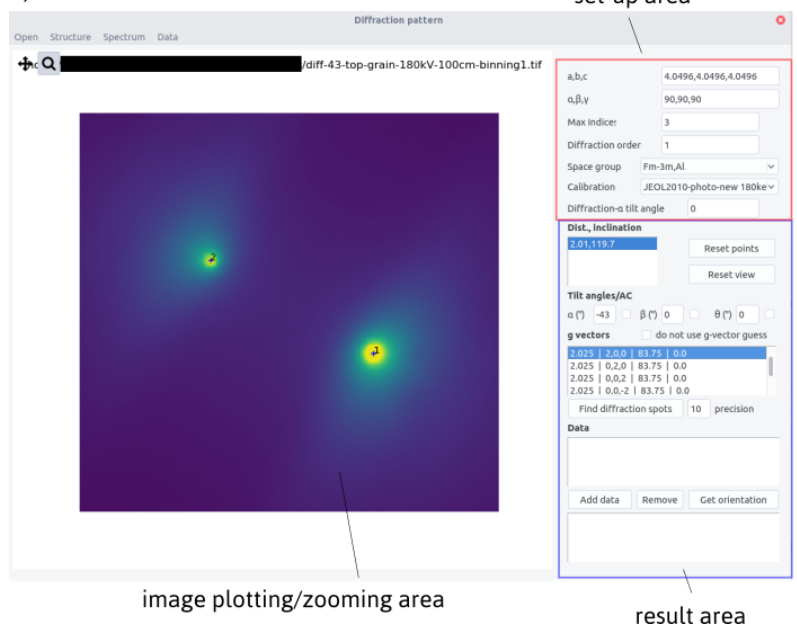
above procedure can then be repeated. corresponding Euler angles.

A second indicator of accuracy is mean d-spacing deviation: they are listed by ascending values of $\langle\Delta \theta\rangle$ and $\langle\Delta d\rangle$. b)

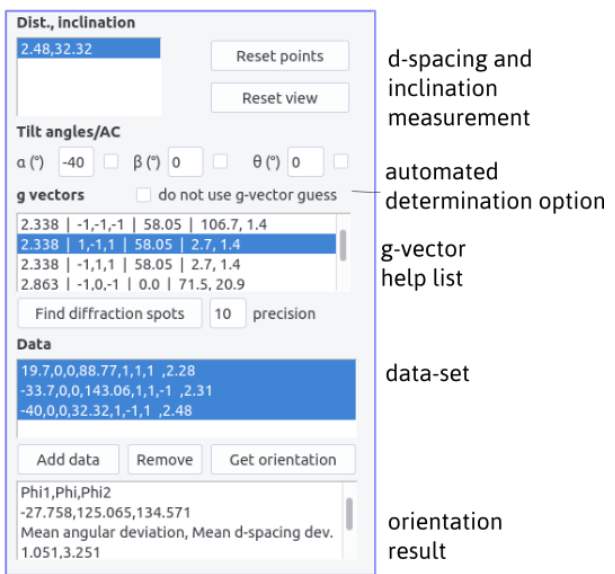

FIGURE 5 a) The graphical user interface of the diffraction tool. b) Zoom of the result panel.

intensities and the angles from the already chosen ones. The data set including the tilt angles are then saved, and the

Once at least 2 data sets $(h, k, l, \alpha, \beta, \eta)$ are given, the orientation can be computed as explained in sec. 2.4. The output window gives the Euler angles $\left(\varphi_{1}, \phi, \varphi_{2}\right)$. When using a fully automated procedure, the user simply needs to collect the $\eta$ and tilt angles. The results window will then propose a set of consistent diffraction vectors and the

As the true orientation is not known, Eq. 9 cannot be derived. Instead the accuracy has to be estimated with the internal consistency of inputs, i.e., the mean orientation deviation $\langle\Delta \theta\rangle$ of all the diffraction vectors:

$$
\langle\Delta \theta\rangle=\frac{1}{N} \sum_{r}^{N} \arccos \left(\left(x_{r}, y_{r}, z_{r}\right)_{\mathrm{M}} \boldsymbol{R}\left(\boldsymbol{D}^{-1}\right)^{\top}\left(h_{r}, k_{r}, I_{r}\right)_{\mathrm{C}}^{\top}\right)
$$

$$
\langle\Delta d\rangle=\frac{1}{N} \sum_{r}^{N} \frac{\left|d_{h_{r} k_{r} l_{r}}-d_{r}\right|}{d_{h_{r} k_{r} I_{r}}}
$$

These two values should be as minimum as possible. Usually a good fit can be obtained with $\langle\Delta \theta\rangle$ below 1 degree (fig. $5 \mathrm{~b}$ ) and $\langle\Delta d\rangle$ of the order of a few percents. In the case where multiple sets of diffraction vectors are proposed,

a)

The approach proposed in sec. 3.1.1 requires at least two g-vectors, either from diffraction pattern series or from one zone axis pattern. However if the diffraction vectors are co-planar, there could be more than one legal result, since spot patterns always have 2 -fold rotation symmetry regardless of the crystal lattice. In pycotem, this $180^{\circ}$ ambiguity is detected and two possible sets of Euler angles are given. Obviously multiple inputs often drastically lower, or even 
eliminate this ambiguity. A set of two-beam diffraction patterns can be downloaded and tested as example in the Supplementary materials.

\subsection{2 | from a Kikuchi pattern}

The tool kikuchi allows to determine the orientation from a Kikuchi pattern with the method described above. Figure 6 shows the user interface, displaying a pattern recorded from bcc iron (available in the Supplementary materials). After setting up the crystal and calibration, at least 2 bands need to be marked. Each band is defined by three points: two on one edge and a third on the other edge. The pattern can be zoomed in to locate the exact position of these points, leading to $\eta$ measurements with a typical error fewer than 1 degree. The crystal orientation can then be retrieved from these bands once the pattern center is defined.

With the derived orientation, the theoretical position of bands can be calculated and displayed in overlay. One may check the accuracy and sensitivity of results by rotating the crystal and tuning camera length and acceleration voltage. The orientation, $\langle\Delta \theta\rangle$ and $\langle\Delta d\rangle$ are recalculated on change of camera length and acceleration voltage. Hence the optimized orientation can be obtained when $\langle\Delta \theta\rangle$ and $\langle\Delta d\rangle$ reach their minimum. The orientation at zero tilt can also be retrieved using Eq. 4 with known tilt angles.

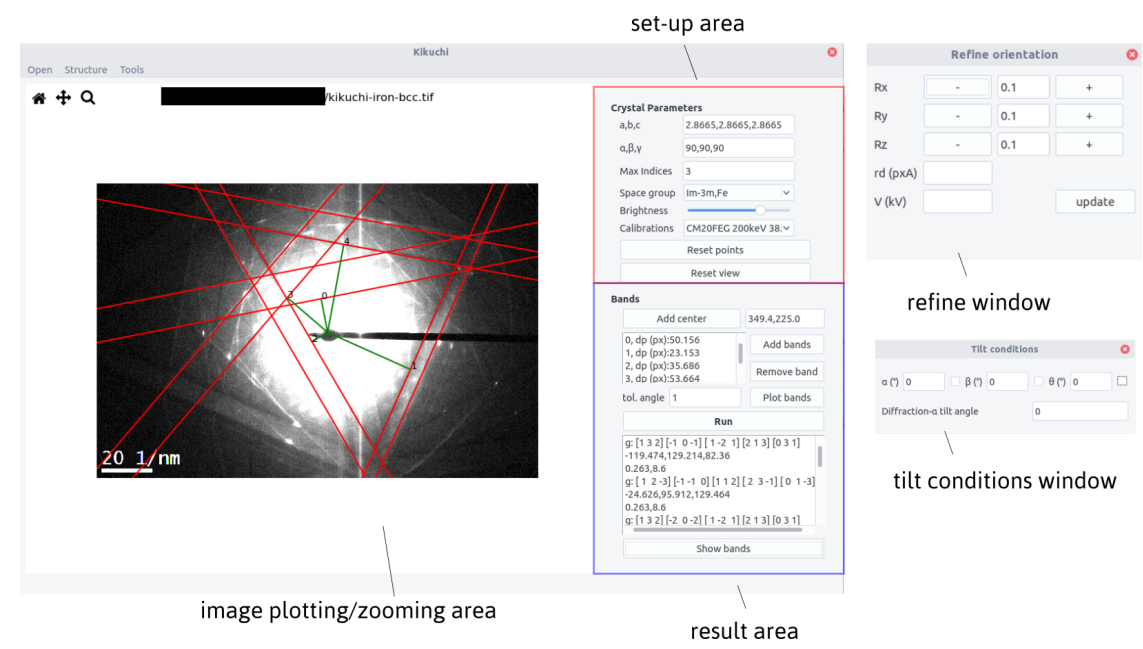

FIGURE 6 The graphical user interface of the kikuchi tool.

\section{2 | Working with crystal orientation}

\subsubsection{Analyzing Burgers vectors by navigating in the reciprocal space}

As an illustrative example, we present analyses performed from a macroscopic bicrystal grown with an expected tilt grain boundary of $\Sigma 7\langle 111\rangle 37.21^{\circ}$. The bicrystal was prepared to electron transparency by FIB using a Xe-source to produce a sample with the $\langle 111\rangle$ rotation axis perpendicular to the foil surface. The two grains were oriented using the 4 diffraction patterns in each grains. The four diffraction patterns and the corresponding BF images are shown for the crystal 1 in Figure 7. The diffraction data used are indicated in table 1. The retrieved Euler angles of crystal 
1 and 2 are $(4.486,54.161,-45.564)_{1}$ and $(-146.939,125.402,-44.689)_{2}$, and the mean orientation deviation angles are $1.149^{\circ}$ and $0.790^{\circ}$, respectively.

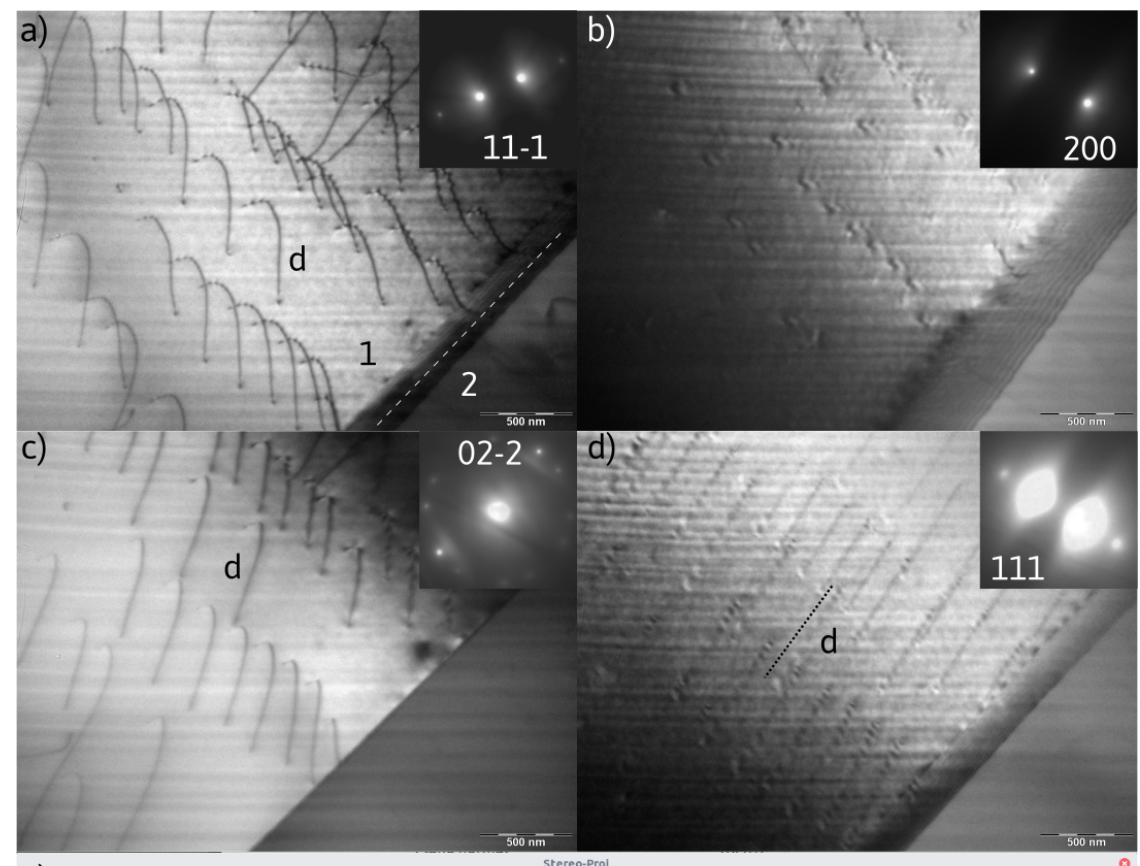

e)

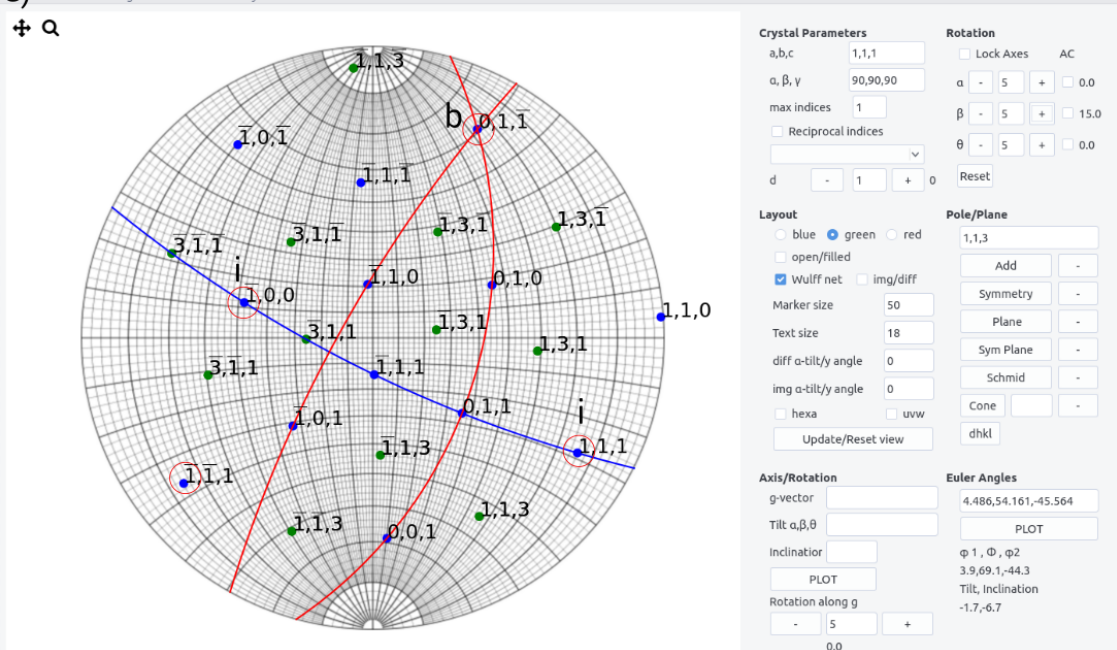

FIGURE 7 Series of BF images obtained at different tilting conditions showing the contrast of dislocations and their shape. In b) and c) the dislocations are hardly visible. The analysis of the images allow to determine the line joining both ends of dislocation $d$ and its Burgers vector. e) is the stereographic projection of the crystal 1 at $(\alpha, \beta)=\left(0^{\circ}, 15^{\circ}\right)$. The g-vectors are encircled and invisibility conditions (i) are indicated. The Burgers vector (b) is at the intersection of the two red planes. The blue planes visually indicate the locus of extinction conditions. 


\begin{tabular}{|c|c|c|c|}
\hline Grain & $\alpha\left(^{\circ}\right)$ & $\eta\left(^{\circ}\right)$ & $(h, k, l)$ \\
\hline 1 & -43.0 & 119.16 & $(2,0,0)$ \\
\hline & 24.0 & 121.83 & $(1,1,1)$ \\
\hline & -1.3 & 34.4 & $(0,2,-2)$ \\
\hline & -21.0 & 65.77 & $(1,1,-1)$ \\
\hline 2 & 19.7 & 87.63 & $(1,1,1)$ \\
\hline & -40.0 & 32.71 & $(1,1,-1)$ \\
\hline & -35.0 & 87.27 & $(0,2,0)$ \\
\hline & -33.7 & 143.24 & $(-1,1,1)$ \\
\hline
\end{tabular}

TABLE 1 Data used for orienting the bicrystal

When crystal orientation is retrieved, stereographic projection can be plotted from the Euler angles. stereoproj can be used to display crystal features, i.e. planes, directions, and to navigate in the direct or reciprocal spaces. The reader can refer to the documentation for a full description of the features. Reaching a given diffraction condition can be easily checked by tilting the crystal until the diffraction vector intersects the equatorial great circle.

As an example, the stereographic projection for crystal 1 is shown in Fig. 7e at $(\alpha, \beta)=\left(0^{\circ}, 15^{\circ}\right)$. In this configuration, the user needs to tilt of an angle $\alpha=-20.5^{\circ}$ to get $\boldsymbol{g}=(2,0,2)$. Alternatively, without relying on manual tilting operations and graphical measurements on the Wulff net, the $\alpha$ angle needed to reach a given diffraction vector can also be automatically retrieved from a $\left(\varphi_{1}, \phi, \varphi_{2}\right)$ orientation, for a single tilt holder or for a double tilt/tilt-rotation holder given a specific $\beta / \theta$ angle.

Using a tilt series, the Burgers vector of the dislocations visible in crystal 1 can now be easily determined. In figure 7(a-d), the four BF images show different dislocation contrasts. Weak visibility is noticed for $\boldsymbol{g}=(2,0,0)$ and $(1,1,1)$, indicating, using the invisibility criterion $\boldsymbol{g} \cdot \boldsymbol{b}=0$, that $\boldsymbol{b}$ is parallel to $[0, \overline{1}, 1]$, i.e. in fcc Al, $\boldsymbol{b}=\boldsymbol{a} / 2[0, \overline{1}, 1]$. Figure $7 \mathrm{e}$ shows that the Burgers vector is at the intersection of the diffracting planes $(2,0,0)$ and $(1,1,1)$. Graphically, the locus of the extinction conditions can be plotted as the plane normal to $b$. It is easy to check when plotting the $\{1,1,3\}$ poles (in green), that an additional extinction should be found for $\boldsymbol{g}=(3,1,1)$ at $(\alpha, \beta)=\left(-17.6^{\circ}, 15^{\circ}\right)$.

\subsection{2 | Determining the misorientation of a bicrystal}

If the orientation determination procedure is performed for two phases or in two grains, the orientation relationship can be defined. The tool misorientation, as a complementary tool to stereoproj, is able to plot two crystals in overlay while rotating them independently. If the same structure is selected for the two grains, the misorientation can be retrieved as being:

$$
M=\boldsymbol{R}_{1} \boldsymbol{R}_{2}^{\top}
$$

where $\boldsymbol{R}_{1}$ and $\boldsymbol{R}_{2}$ are the orientation matrices.

As $\boldsymbol{M}$ is a rotation matrix, one could extract the couple angle-axis, $(\theta, \boldsymbol{u})$ according to: 


$$
\theta=\arccos \left(\frac{\operatorname{tr}(\boldsymbol{M})-1}{2}\right), \boldsymbol{M u}=\boldsymbol{u}
$$

\section{a)}

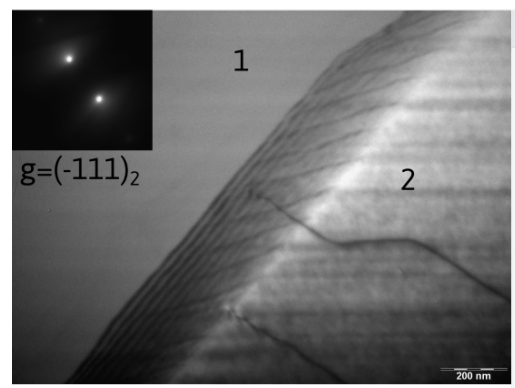
grains, 1 in blue and 2 in red.

$$
M=R_{1} S R_{2}^{-1}
$$

where $S$ is one of symmetry operation matrices. Hence for a cubic crystal, there are 24 equivalent misorientations.

Figure $8 \mathrm{a}$ shows a BF image of the bicrystal inclined and $8 \mathrm{~b}$ the superimposed stereographic projections of two

a) b)

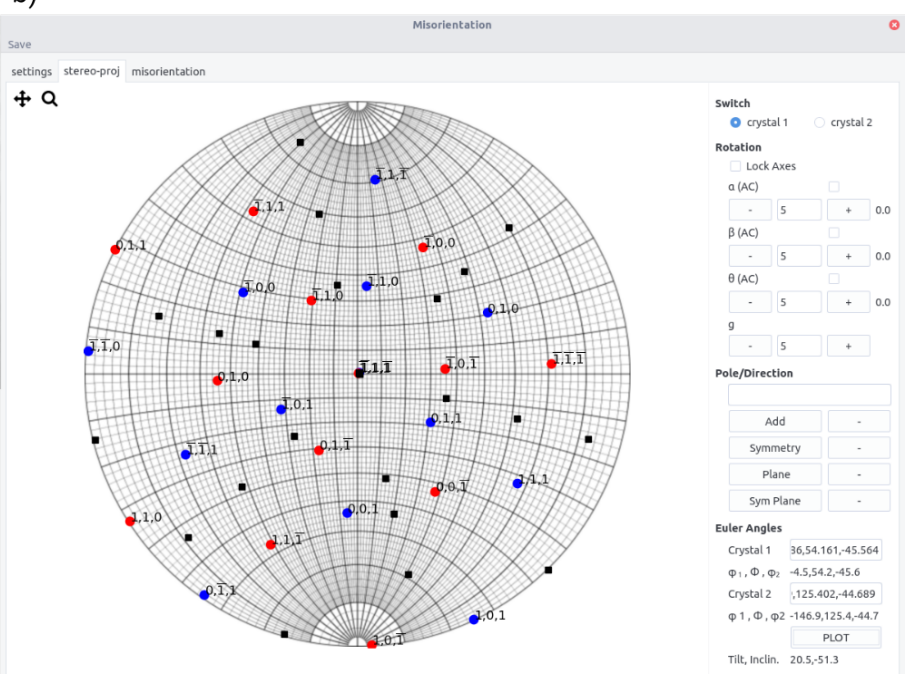

FIGURE 8 a) BF of the interface between grain 1 and 2 in an Al bicrystal. b) Misorientation shown by the superimposed stereographic projections. In brown are shown the 24 misorientation axes.

The 24 misorientation axes are shown in brown in fig. 8b. The lists of axes and the angles are also obtained. The minimum angle is $37.7^{\circ}$ along $[56,57,58]_{1}$. The results is close to a $\Sigma 7\langle 111\rangle 37.21^{\circ}$. The deviation can be easily obtained by rotating the crystal 1 by $37.21^{\circ}$ along [111 $]_{1}$ and computing the misorientation. It gives a minimum misorientation angle of $0.67^{\circ}$ along $[\overline{9}, 17,97]_{1}$ inclined about $44^{\circ}$ from $[\overline{1} 11]_{1}$.

\section{3 | Interface determination and slip plane analysis}

Using the interface tool (fig. 9), we determined the interface plane using measurements at 4 diffraction conditions. Since the algorithm can work with multiple inputs, we perform slightly different measurements several times at each diffraction condition. This procedure takes into account the uncertainty on the trace directions and projection widths. 
a)
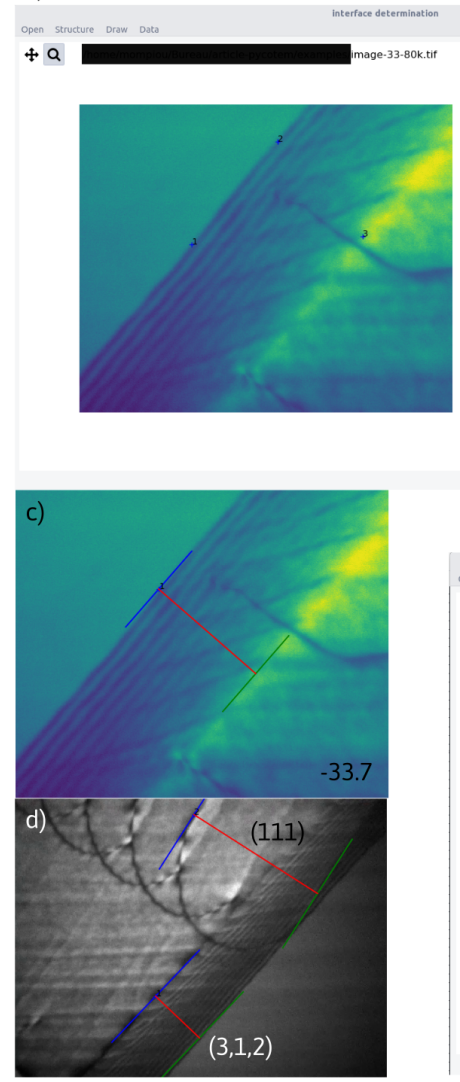

b)
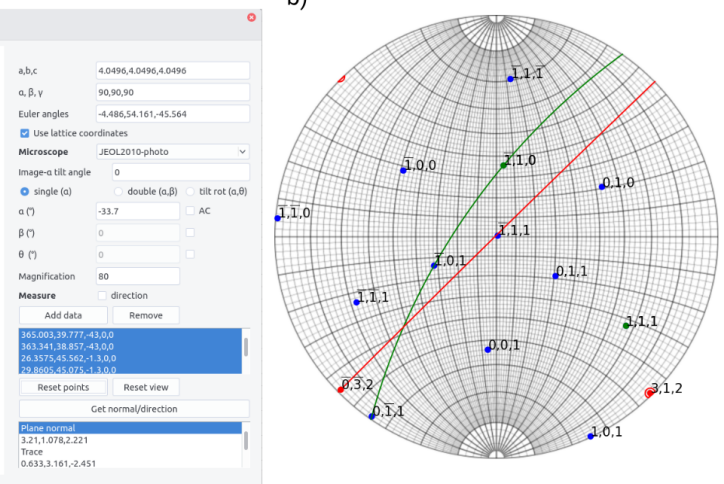

e)

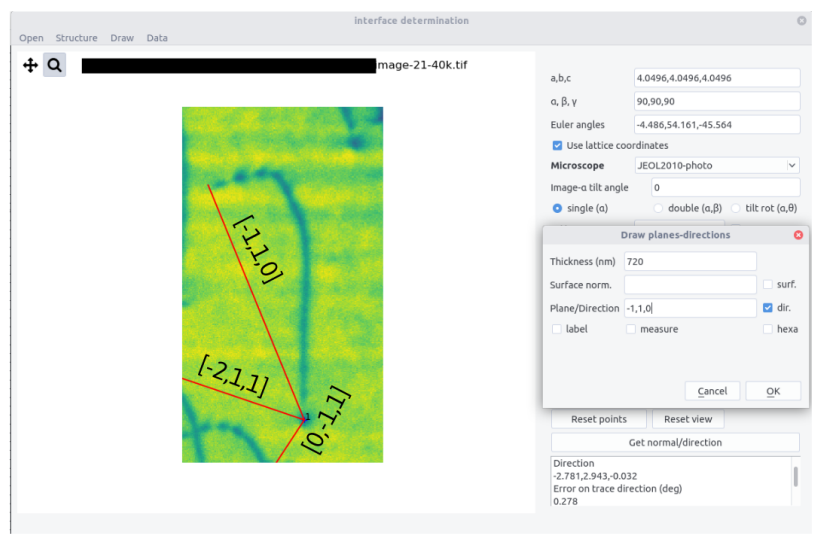

FIGURE 9 Interface tool. a) General view b) Stereographic projection of grain 1, with the grain boundary plane $(3,1,2)$ in red, the slip plane $(1,1,1)$ in green. In red is also shown the trace direction $[0, \overline{3}, 2]$ and in green the line direction $[\overline{1}, 1,0] . c)$ - e) show the drawing functionality for plane and line direction.

The data set $\left(\alpha_{i}, w_{i}, \eta_{i}\right)$ are shown in table 2. Redundant information are used owing uncertainty in determining the trace width and direction angle. This leads to an interface plane $(3.21,1.078,2.221)$, with a $92 \%$ confidence in a $1.7^{\circ}$ interval. The trace direction $[0.633,3.161,-2.451]$ is almost perpendicular to the beam direction at zero tilt. Although the sample surface $s$ cannot be retrieved, this however indicates that it should also be probably parallel to the beam direction at zero tilt. Thus the sample thickness can be estimated from: $t=d \sqrt{1-(\boldsymbol{n} \cdot \boldsymbol{s})^{2}} \approx 645 \pm 60 \mathrm{~nm}$.

The line direction joining the two ends of the dislocation $d$ in crystal 1 (fig. 7) was also determined using the data set in table 3. Here again multiple inputs were used and leads to [-2.781, 2.943, -0.032$]$ within a confidence interval of $0.3^{\circ}$. This direction is thus close to $[\overline{1}, 1,0]$.

The results of grain boundary plane, dislocation line direction, and slip plane are checked by visualizing them on the image with the drawing feature. A good agreement was found for the plane (fig. 9c) and line direction (fig. 9d) 


\begin{tabular}{|c|c|c|}
\hline$\alpha\left(^{\circ}\right)$ & $w(\mathrm{~nm})$ & $\eta\left(^{\circ}\right)$ \\
\hline-43 & 365.003 & 39.777 \\
\hline & 363.341 & 38.857 \\
\hline-33.7 & 241.824 & 39.405 \\
\hline & 247.568 & 39.775 \\
\hline-21 & 190.090 & 45.503 \\
\hline & 201.471 & 43.452 \\
\hline-1.3 & 26.357 & 45.562 \\
\hline & 29.860 & 45.075 \\
\hline 24 & 200.553 & 44.293 \\
\hline & 193.741 & 44.846 \\
\hline
\end{tabular}

TABLE 2 Data used for determining the grain boundary plane

\begin{tabular}{|c|c|c|}
\hline$\alpha\left(^{\circ}\right)$ & $I(\mathrm{~nm})$ & $\left.\eta l^{\circ}\right)$ \\
\hline-21 & 549.21 & -19.97 \\
\hline & 552.548 & -20.01 \\
\hline & 551.843 & -19.877 \\
\hline-1.3 & 553.008 & -19.394 \\
\hline & 526.585 & 6.293 \\
\hline & 526.784 & 6.217 \\
\hline & 527.537 & 6.026 \\
\hline 24 & 639.271 & 34.424 \\
\hline & 647.456 & 34.38 \\
\hline & 641.594 & 34.391 \\
\hline & 635.692 & 34.292 \\
\hline
\end{tabular}

TABLE 3 Data used for determining line direction

using foil thickness of 645 and $720 \mathrm{~nm}$, respectively. The projected traces of the $(1,1,1)$ plane also agree to the traces left by a gliding dislocation during an in-situ tensile test as shown in fig. $9 \mathrm{~d}$. Knowing that $\boldsymbol{b}=a / 2[0, \overline{1}, 1]$, from sec. 3.2.1, the slip plane, defined as $\boldsymbol{n}_{s}=\boldsymbol{b} \times \boldsymbol{l}=(1,1,1)$, is indeed in agreement with this result. 


\section{SUMMARY AND CONCLUSIONS}

We have presented a series of software tools that can be easily set-up to help TEM users to retrieve the crystallographic features of defects (dislocations, interfaces). The newly developed method to determine crystal orientations is based on a least-squares method and allows to evaluate experimental errors. As an interesting result, we demonstrate, that even when using slightly erroneous data, both orientation and plane determination can be retrieved with a good accuracy if data are numerous and redundant. The workflow consists first in determining the orientation either from a series of two beam diffraction patterns or from a Kikuchi pattern. While the latter is intrinsically more accurate, within few tenths of a degree, the first one is convenient for users who also want to analyze, for instance, dislocation Burgers vectors. Along with tilt series, the user can exploit BF/DF images taken at different tilt to determine interface plane also with a good accuracy. The workflow proposed can be set up rapidly either for post-processing or inline at the microscope. This is valuable especially during in-situ experiments where some analyses must be performed on site and are no longer possible afterwards.

\section{acknowledgements}

We are grateful to D. Molodov at RWTH, Aachen for bicrystal growth, F. Mialhes and H. Chauvin at CNES, Toulouse for sample preparation and J. Du at Tsinghua Univ, Beijing, for fruitful discussions.

\section{5 | APPENDIX}

The rotation matrices used are:

$$
\begin{aligned}
& R_{x}(\theta)=\left(\begin{array}{ccc}
1 & 0 & 0 \\
0 & \cos \theta & -\sin \theta \\
0 & \sin \theta & \cos \theta
\end{array}\right) \\
& R_{y}(\theta)=\left(\begin{array}{ccc}
\cos \theta & 0 & \sin \theta \\
0 & 1 & 0 \\
-\sin \theta & 0 & \cos \theta
\end{array}\right) \\
& R_{z}(\theta)=\left(\begin{array}{ccc}
\cos \theta & -\sin \theta & 0 \\
\sin \theta & \cos \theta & 0 \\
0 & 0 & 1
\end{array}\right)
\end{aligned}
$$

\section{references}

[1] J. W. Edington. Electron Diffraction in the Electron Microscope, pages 1-77. Macmillan Education UK, London, 1975.

[2] David B. Williams and C. Barry Carter. Indexing Diffraction Patterns, pages 265-287. Springer US, Boston, MA, 1996.

[3] D.R.G. Mitchell. Difftools: Electron diffraction software tools for digitalmicrograph. Microscopy Research and Technique, 71(8):588-593, 2008.

[4] C.H. Wu, W.T. Reynolds, and M. Murayama. A software tool for automatic analysis of selected area diffraction patterns within digital micrograph ${ }^{\mathrm{TM}}$. Ultramicroscopy, 112(1):10 - 14, 2012.

[5] Miloslav Klinger and Aleš Jäger. Crystallographic Tool Box (CrysTBox): automated tools for transmission electron microscopists and crystallographers. Journal of Applied Crystallography, 48(6):2012-2018, Dec 2015. 
[6] P.A. Stadelmann. Ems - a software package for electron diffraction analysis and hrem image simulation in materials science. Ultramicroscopy, 21(2):131 - 145, 1987.

[7] C. Boudias and D. Monceau. Carine crystallography. version 3.1, 1998. CaRIne Crystallography, Senlis, France.

[8] X. Z. Li. Landyne - a software suite for electron diffraction simulation and analysis. Microscopy and Microanalysis, 22(S3):564-565, 2016.

[9] 2020. CrystalMaker Software Ltd, Begbroke, UK.

[10] Stefan Zaefferer. New developments of computer-aided crystallographic analysis in transmission electron microscopy. Journal of Applied Crystallography, 33(1):10-25, 2000.

[11] J.-J. Fundenberger, A. Morawiec, E. Bouzy, and J.S. Lecomte. Polycrystal orientation maps from tem. Ultramicroscopy, 96(2):127 - 137, 2003.

[12] Guilin Wu and Stefan Zaefferer. Advances in tem orientation microscopy by combination of dark-field conical scanning and improved image matching. Ultramicroscopy, 109(11):1317 - 1325, 2009.

[13] A. Valey, E.F. Rauch, L. Clément, and F. Lorut. Retrieving overlapping crystals information from tem nano-beam electron diffraction patterns. Journal of Microscopy, 268(2):208-218, 2017.

[14] D. Viladot, M. Véron, M. Gemmi, F. Peiro, J. Portillo, S. Estradé, J. Mendoza, N. Llorca-Isern, and S. Nicopoulos. Orientation and phase mapping in the transmission electron microscope using precession-assisted diffraction spot recognition: state-of-the-art results. Journal of Microscopy, 252(1):23-34, 2013.

[15] Rui-Xun Xie and Wen-Zheng Zhang. tompas: a free and integrated tool for online crystallographic analysis in transmission electron microscopy. Journal of Applied Crystallography, 53(2), Apr 2020.

[16] T. Duden, A. Gautam, and U. Dahmen. Kspacenavigator as a tool for computer-assisted sample tilting in high-resolution imaging, tomography and defect analysis. Ultramicroscopy, 111(11):1574 - 1580, 2011.

[17] N. Cautaerts, R. Delville, and D. Schryvers. Alphabeta: a dedicated open-source tool for calculating tem stage tilt angles. Journal of Microscopy, 273(3):189-198, 2019.

[18] X.-Z. Li. SPICA: stereographic projection for interactive crystallographic analysis. Journal of Applied Crystallography, 49(5):1818-1826, Oct 2016.

[19] X.-F. Gu, T. Furuhara, and W.-Z. Zhang. PTCLab: free and open-source software for calculating phase transformation crystallography. Journal of Applied Crystallography, 49(3):1099-1106, Jun 2016.

[20] Frédéric Mompiou, Marc Legros, and Sylvie Lartigue-Korinek. Deformation mechanisms in submicron be wires. Journal of Materials Research, 32(24):4616-4625, 2017.

[21] Dong Qiu and Mingxing Zhang. A simple and inclusive method to determine the habit plane in transmission electron microscope based on accurate measurement of foil thickness. Materials Characterization, 94:1-6, 2014.

[22] M. X. Zhang, P. M. Kelly, and J. D. Gates. Determination of habit planes using trace widths in tem. Materials Characterization, 43(1):11-20, 1999.

[23] R.-X. Xie, M. Larranaga, F. Mompiou, and W.-Z. Combe, N.and Zhang. A general and robust analytical method for interface normal determination in tem. Ultramicroscopy, page 113009, 2020.

[24] 2020. https://mompiou.github.io/pycotem/.

[25] H.-J. Bunge. 2 - orientation of individual crystallites. In H.-J. Bunge, editor, Texture Analysis in Materials Science, pages 3 - 41. Butterworth-Heinemann, 1982. 
371

372

373

374

375

376

377

[26] J. K. Mackenzie. The estimation of an orientation relationship. Acta Crystallographica, 10(1):61-62, Jan 1957.

[27] B. K. P. Horn. Closed-form solution of absolute orientation using unit quaternions. Journal of the Optical Society of America a-Optics Image Science and Vision, 4(4):629-642, 1987.

[28] B. Efron. Bootstrap methods: Another look at the jackknife. Ann. Statist., 7(1):1-26, 011979.

[29] 2020. https://github.com/mompiou/pycotem/ and https://pypi.org/project/pycotem/.

\section{GRAPHICAL ABSTRACT}

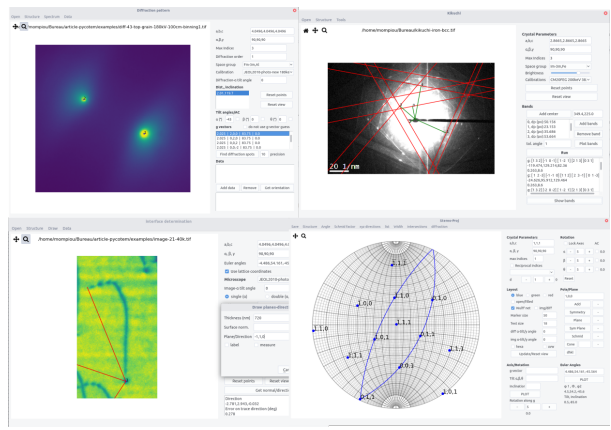

